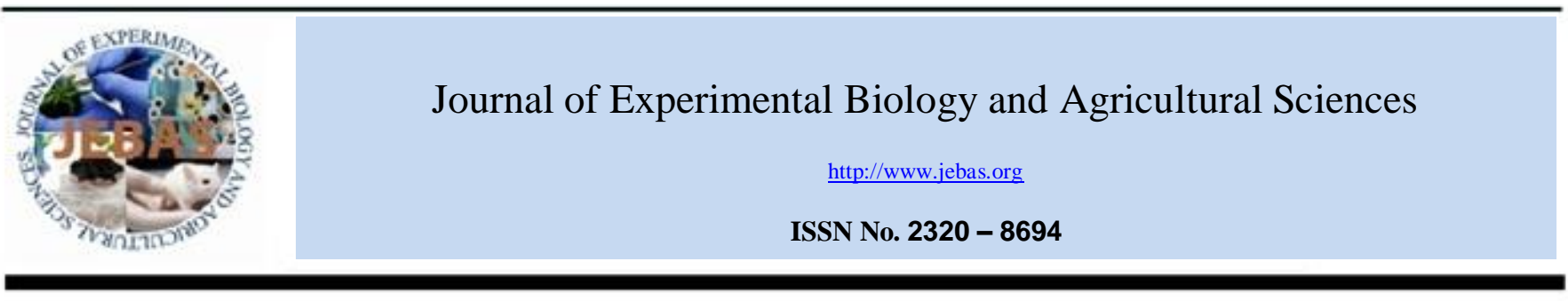

\title{
EFFECT OF NITROGEN FERTILIZATION ON SOYBEAN PRODUCTION UNDER TWO CROPPING PATTERNS
}

\section{Suryantini and Henny Kuntyastuti}

Indonesian Legume and Tuber Crops Institute (ILETRI)

Received - March 25, 2015; Revision - April 28, 2015; Accepted - June 29, 2015

Available Online - July 05, 2015

DOI: http://dx.doi.org/10.18006/2015.3(3).316.323

\section{KEYWORDS \\ Soybean \\ Nitrogen fertilizer \\ Cropping pattern \\ Grain yield}

\begin{abstract}
Response of soybean crop toward $\mathrm{N}$ fertilizer in paddy fields is influenced by the cropping pattern. Present study was conducted for finding out the effect of two cropping pattern viz rice-rice-soybean and rice-soybean-soybean and $\mathrm{N}$ fertilization on the production of soybean crops. Results of the study revealed that the cultivation of soybean after rice required higher amount of $\mathrm{N}$ fertilizer $(30 \mathrm{~kg} \mathrm{~N} / \mathrm{ha}$ of urea or $90 \mathrm{~kg} \mathrm{~N} /$ ha of ZA) to increase the grain yield from $0.5 \mathrm{t} / \mathrm{ha}$ (control) to $3.4 \mathrm{t} /$ ha while in case of soybean after soybean cropping pattern soybean did not required any additional dose of $\mathrm{N}$ fertilizer for increasing the grain yield up to $3 \mathrm{t} / \mathrm{ha}$.
\end{abstract}

* Corresponding author

E-mail: suryantinibalitkabi@yahoo.com (Suryantini)

Peer review under responsibility of Journal of Experimental Biology and Agricultural Sciences.

Production and Hosting by Horizon Publisher (www.myvision.webs.com/horizon.html)

All rights reserved.
All the article published by Journal of Experimental Biology and Agricultural Sciences is licensed under a Creative Commons Attribution-NonCommercial 4.0 International License Based on a work at www.jebas.org.

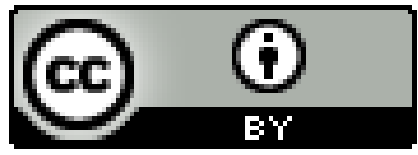




\section{Introduction}

Soil nitrogen plays a crucial role in sustainable agricultural production but it can be disturbed by disturbing the supply of useable nitrogen (Santoso et al., 2001; Spiertz, 2010). It has been well reported that Indonesian paddy soil is generaly deficient of N nutrient. According to Adisarwanto et al. (1998) paddy soil of East Java, Bali and NTB provinces have very low $\mathrm{N}$ content $(0.07$ to $0.25 \%)$. Similarly, in another survey Adisarwanto et al. (1999) reported that paddy soil of Ngawi and Banyuwangi have $\mathrm{N}$ status between 0.12 to $0.36 \%$. Furthermore, paddy soil of Mojokerto, Banyuwangi and NTB have Entisol type of soil and showed $\mathrm{NO}_{3}$ concentration range between $5-70 \mathrm{mg} / \mathrm{kg}$ and $\mathrm{N}$ content between 0.08 to $0.19 \%$ (Adisarwanto \& Suhartina, 2000; Adisarwanto et al., 2001). Soil texture affects the rate of $\mathrm{N}_{2} \mathrm{O}$ losses, coarse-texture soil favor the loss of $\mathrm{N}_{2} \mathrm{O}$ while fine-texture soil have capacity to retain the $\mathrm{N}_{2} \mathrm{O}$ and decrease the loss of Nitrogen from the soil (Gentile et al., 2008). The decline in the intensity of tillage accompanied by an increase in crop diversification is very effective to increase the levels of total soil N (Al Kaisi et al., 2005). According to Dou et al. (2007) continuous cultivation soybean of had negative effect on the soil $\mathrm{N}$ level as compared to the sorghum-wheat-soybean and wheat-soybean cropping pattern.

Total $\mathrm{N}$ uptake by a plant also depends on the various environmental factor like types of soil, soil nature, total available nitrogen in soil and cropping pattern. Nature of soil and dose of fertilizers severely affect the fertilization process, according to Adisarwanto et al. (1998) urea is not a good nitrogen source for the Vertisol or Entisol paddy soil. It was reported that fertilization by urea @ $50 \mathrm{~kg} / \mathrm{ha}$ did not increase soybean grain yield in Vertisol paddy field at Ngawi or Entisols at Jambegede and Banyuwangi (Adisarwanto et al. 1998). Even at the application of $100 \mathrm{~kg}$ urea/ha on soybean followed by soybean (rice-soybean-soybean) and soybean followed by rice (rice-rice-soybean) also did not increase the grain yield (Suryantini \& Kuntyastuti, 1998). However, the application of ZA at $50 \mathrm{~kg} / \mathrm{ha}$ increased soybean grain yield by $0.26 \mathrm{t} / \mathrm{ha}(18 \%)$ in Entisol at Jambegede and by $0.36 \mathrm{t} / \mathrm{ha}$ $(19 \%)$ in Vertisol at Ngawi (Riwanodja et al., 2001). Furthermore, the combination of $50 \mathrm{~kg} / \mathrm{ha}$ urea and Selementary $150 \mathrm{~kg} / \mathrm{ha}$ also increased grain yield by $0.81 \mathrm{t} / \mathrm{ha}$ (86\%) (Adisarwanto et al. 2001).

Similarly, Suryantini (2008) reported that the application of 50 $\mathrm{kg} / \mathrm{ha}$ urea did not increase grain yield of soybean planted after rice. Conversely, in soybean planted after soybean (ricesoybean-soybean), the application of $50 \mathrm{~kg}$ urea/ha or Rhizobium inoculation at recommended rate increased the grain yield of soybean. Both nitrogen source (urea and Rhizobium) did not increase grain yield of soybean when there was manure residues, or given to soybean planted after rice (Suryantini \& Rahmianna, 2001). Information regarding the effect of cropping pattern on nitrogen fertilization are in scarcity therefore present study have been conducted to find out the effect of two cropping pattern viz rice-rice-soybean and rice-soybean-soybean on the nitrogen fertilization.

\section{Materials and Methods}

The experiment was conducted in Entisol paddy fields at Jambegede Research Station Malang-Indonesia, during the dry seasons of 2010. Soybeans were planted after rice and after soybean crops in the cropping sequences of rice-rice-soybean and rice-soybean-soybean respectively. A randomized block design with three replications was used for present study. $\mathrm{N}$ fertilizer were applied at the rate of $0,30,60,90,120,150,180$ and $210 \mathrm{~kg} \mathrm{~N} /$ ha from two sources, namely Urea and ZA (ammonium sulfate - $\left.\left(\mathrm{NH}_{4}\right)_{2} \mathrm{SO}_{4}\right)$. Sulfur supplement (S) @ $210 \mathrm{~kg} \mathrm{~N} /$ ha was equated to all treatments using sulfur powder. This sulfur was also added in control namely without $\mathrm{N}$ and without $\mathrm{N}$ and $\mathrm{S}$. The sulfur powder containing $85 \% \mathrm{~S}$; while (NH4) 2SO4 containing $21 \% \mathrm{~N}$ and $24 \% \mathrm{~S}$ and urea containing $46 \% \mathrm{~N}$.

A composite soil samples was collect from each site before planting and analyzed for soil physical- chemical analysis (Table 1 and 2). Soil $\mathrm{pH}$ was measured in slurry (1:2.5 soil to water ratio) by glass electrode using an electronic $\mathrm{pH}$, available $\mathrm{P}$ was measured by the method described by Bray 1 . Total available $\mathrm{N}$ before planting was determined using the micro-Kjeldahl method while organic carbon was determined by the Walkley and Black wet oxidation method. Exchangeable bases were determined by ammonium acetate extraction, with $\mathrm{Ca}$ and $\mathrm{Mg}$ estimated by Atomic Absorption Spectrophotometer (AAS), and K by the flame photometer. Zn, $\mathrm{Cu}, \mathrm{Fe}$ and $\mathrm{Mn}$ were measured by extraction using DTPA, with quantitative determination by AAS. Bulk density was determined by the cylinder method and total porosity was calculated from values of bulk and particle density. Clay size fraction was determined by the pipette method. The texture classes were defined according to the textural triangle by FAO.

Willis soybean varieties were planted in plots measuring $4 \mathrm{~m} \mathrm{x}$ $5 \mathrm{~m}$ with a spacing of $40 \mathrm{~cm} \mathrm{x} 10 \mathrm{~cm}$, two plants / hill. Thinning was done at the age of 10 DAP (days after planting) and spared two plants / hill. Fertilizer $\mathrm{N}$ was applied twice (50\% each) at planting and flowering in bands $10 \mathrm{~cm}$ from the plant rows. Weeding was done two times at 15 and 35 DAP, and watered every 20 days. Control of pests / diseases was done by monitoring the control threshold. Plants were harvested when $95 \%$ of pods had turned brown and fallen leaves. Data were collected at 60 DAP from samples of 5 plants per plot for shoot and root dry weight as well as number of branches per plant. Oven dried shoots were grinded homogenously and left at $400^{\circ} \mathrm{C}$ for $1 \mathrm{~h}$ for the development of ash, this ash samples were analyzed for estimating the total mineral including $\mathrm{K}, \mathrm{Ca}, \mathrm{Mg}, \mathrm{P}$, and $\mathrm{S}$. Chemical analysis were done by energy-dispersive $\mathrm{x}$-ray analysis (EDXA). While total $\mathrm{N}$ was determined by modified Kjeldahl digestion colorimetric method. At harvesting time 10 plants per plot were collected 
for measuring plant height, number of filled pods per plant and 100 seed weight, while the harvested plots of $3 \mathrm{~m} \mathrm{x} 4 \mathrm{~m}$ was observed for number of harvested plants (plant population) and grain yield. Statistical analysis of data was carried out using standard analysis of variance (Gomez \& Gomez, 1984). The significance of the treatment effect was determined using the F-test. To determine the significance of the difference between the means of treatments, Duncan Multiple Range Test (DMRT) was computed at the $5 \%$ probability level.

\section{Results and Discussion}

\subsection{Rice-Soybean-Soybean Cropping Pattern}

No significant difference was reported in Entisol paddy soil after rice and soybean cultivation. Soil after rice and soybean crops were poor in organic matter and macro nutrients likes $\mathrm{N}$ and $\mathrm{S}$, but these soils were rich in $\mathrm{P}, \mathrm{K}, \mathrm{Ca}$ and $\mathrm{Mg}$ as well as for other micro nutrients (Table 1). The texture of the studied soil was clay loam and it has $17-19 \%$ water holding capacity and high cation exchange capacity (CEC). The saturated hydraulic conductivity (Ksat) for the studied soil was low (0.8 $\mathrm{cm} /$ hour) and the penetration of these soil was high (Table 2).

Soybean after soybean grew well with a population nearly 460 thousand/ha approaching the optimum population. Optimal plant population is one of the requirements to achieve high yield (Table 4). Though conditions are favourable but fertilization by $\mathrm{N}$ did not have any significatory effect on shoot and root dry weight as well as number of branches. Dry weight of shoot and root reached 9.98 and $0.91 \mathrm{~g} / \mathrm{plant}$ at the application of $180 \mathrm{Kg} / \mathrm{ha}$ and $150 \mathrm{Kg} / \mathrm{ha}$ respectively (totally equivalent to $4.53 \mathrm{t} /$ ha stover). Increasing the dose of urea favor the plant growth characters. Application of urea and ZA $@ 30 \mathrm{~kg} /$ ha have positive effect on the plant growth characters and application at this level increased plant height from 40.3 $\mathrm{cm}$ (control) to 45.7 and $46.3 \mathrm{~cm}$ respectively and this treatments shows superiority over the other treatments of ZA. Further increases the levels of $\mathrm{N}$ and ZA have not showing any significant effect on the plant heights and number of branches/plant (Table 3).

Table 1 Chemical properties of Entisol paddy soil in rice-rice-soybean and rice-soybean-soybean cropping system $(0-20 \mathrm{~cm}$ depth).

\begin{tabular}{|lcc|}
\hline Chemical properties & Rice-Rice-Soybean & Rice-Soybean-Soybean \\
\hline $\mathrm{pH} \mathrm{H} \mathrm{H}_{2}$ & 0.78 & 0.80 \\
\hline $\mathrm{pH} \mathrm{KCl}$ & 1.20 & 1.23 \\
\hline $\mathrm{C}-\mathrm{org}(\%)$ & 2.56 & 2.50 \\
\hline $\mathrm{N}(\%)$ & 53.00 & 51.30 \\
\hline $\mathrm{P} 2 \mathrm{O} 5 \mathrm{Bray} \mathrm{I}(\mathrm{ppm})$ & 399.49 & 399.50 \\
\hline $\mathrm{SO} 4(\mathrm{ppm})$ & 1.84 & 0.80 \\
\hline $\mathrm{K}(\mathrm{me} / 100 \mathrm{~g})$ & 20.4 & 20.8 \\
\hline $\mathrm{Ca}(\mathrm{me} / 100 \mathrm{~g})$ & 0.13 & 0.45 \\
\hline $\mathrm{Mg}(\mathrm{me} / 100 \mathrm{~g})$ & 39.0 & 41.0 \\
\hline $\mathrm{KTK}(\mathrm{me} / 100 \mathrm{~g})$ & 22.0 & 22.0 \\
\hline $\mathrm{Fe}(\mathrm{ppm})$ & 17.0 & 19.0 \\
\hline $\mathrm{Zn}(\mathrm{ppm})$ & 16 & 16 \\
\hline $\mathrm{Cu}(\mathrm{ppm})$ & 44 & 50 \\
\hline $\mathrm{Mn}(\mathrm{ppm})$ & 40 & 34 \\
\hline $\mathrm{D}$ & & \\
\hline
\end{tabular}

Data are mean of three replicates

Table 2. Physical properties of Entisol paddy soil used for rice-rice-soybean and rice-soybean-soybean cropping system $(0-20 \mathrm{~cm}$ depth).

\begin{tabular}{|l|c|c|}
\hline Physical properties & Rice-Rice-Soybean & Rice-Soybean-Soybean \\
\hline Ksat $(\mathrm{cm} /$ hour$)$ & 0.78 & 0.80 \\
\hline Bulk density $\left(\mathrm{g} / \mathrm{cm}^{3}\right)$ & 1.20 & 51.23 \\
\hline Porosity $(\%)$ & 53.00 & 399.50 \\
\hline Penetration $\left(\mathrm{N} / \mathrm{cm}^{2}\right)$ & 399.49 & 41.0 \\
\hline Water content $\mathrm{pF} 2,5(\%)$ & 39.0 & 22.0 \\
\hline Water content $\mathrm{pF} 4,2(\%)$ & 22.0 & 19.0 \\
\hline Available water $(\%)$ & 17.0 & 16 \\
\hline Sand $(\%)$ & 16 & 50 \\
\hline Silt $(\%)$ & 44 & 34 \\
\hline Clay $(\%)$ & 40 & Clay loam \\
\hline Texture class & Clay loam & \\
\hline
\end{tabular}

Data are mean of three replicates 
Table 3 Effect of $\mathrm{N}$ fertilizer on vegetative growth of soybean in rice-soybean-soybean cropping system.

\begin{tabular}{|c|c|c|c|c|}
\hline \multirow[t]{2}{*}{ Nitrogen Source and its dose } & \multicolumn{2}{|c|}{ Dry weight (g/plant) } & \multirow[t]{2}{*}{ Plant height (cm) } & \multirow{2}{*}{$\begin{array}{l}\text { Branches } \\
\text { (no./plant) }\end{array}$} \\
\hline & Shoot & Root & & \\
\hline Without NS (Control) & $8.56 \pm 0.91^{\mathrm{c}}$ & $0.80 \pm 0.02^{\mathrm{b}}$ & $43.2 \pm 1.56^{\mathrm{d}}$ & 2.0 \\
\hline Without N (Control) & $8.98 \pm 0.73^{\mathrm{bc}}$ & $0.76 \pm 0.10^{b}$ & $40.3 \pm 0.97^{\mathrm{f}}$ & 1.9 \\
\hline Urea $-30 \mathrm{Kg} \mathrm{N} / \mathrm{ha}$ & $8.82 \pm 1.02^{\mathrm{bc}}$ & $0.82 \pm 0.08^{\mathrm{ab}}$ & $45.7 \pm 1.56^{\mathrm{ab}}$ & 1.4 \\
\hline Urea $-60 \mathrm{Kg} \mathrm{N} / \mathrm{ha}$ & $9.56 \pm 0.56^{\mathrm{b}}$ & $0.90 \pm 0.12^{\mathrm{a}}$ & $45.3 \pm 2.53^{\mathrm{b}}$ & 2.3 \\
\hline Urea $-90 \mathrm{Kg} \mathrm{N} / \mathrm{ha}$ & $9.71 \pm 0.85^{\mathrm{ab}}$ & $0.87 \pm 0.05^{\mathrm{ab}}$ & $44.1 \pm 1.00^{\mathrm{c}}$ & 1.4 \\
\hline Urea $-120 \mathrm{Kg} \mathrm{N} / \mathrm{ha}$ & $9.73 \pm 0.99^{\mathrm{ab}}$ & $0.72 \pm 0.06^{\mathrm{bc}}$ & $45.1 \pm 0.87^{\mathrm{b}}$ & 2.0 \\
\hline Urea $-150 \mathrm{Kg}$ N/ha & $9.64 \pm 0.35^{\mathrm{ab}}$ & $0.91 \pm 0.10^{\mathrm{a}}$ & $44.4 \pm 0.99^{\mathrm{bc}}$ & 1.7 \\
\hline Urea $-180 \mathrm{Kg} \mathrm{N} / \mathrm{ha}$ & $9.98 \pm 0.56^{\mathrm{ab}}$ & $0.73 \pm 0.09^{\mathrm{bc}}$ & $42.7 \pm 1.21^{\mathrm{de}}$ & 2.3 \\
\hline Urea $-210 \mathrm{Kg} \mathrm{N} / \mathrm{ha}$ & $9.12 \pm 0.79^{\mathrm{b}}$ & $0.64 \pm 0.05^{\mathrm{c}}$ & $44.1 \pm 1.90^{\mathrm{c}}$ & 2.5 \\
\hline ZA -30 Kg N/ha & $10.47 \pm 1.1^{\mathrm{a}}$ & $0.73 \pm 0.07^{\mathrm{bc}}$ & $46.3 \pm 1.28^{\mathrm{a}}$ & 1.8 \\
\hline ZA -60 Kg N/ha & $9.68 \pm 0.15^{\mathrm{ab}}$ & $0.89 \pm 0.02^{\mathrm{a}}$ & $45.0 \pm 2.90^{\mathrm{b}}$ & 2.0 \\
\hline ZA $-90 \mathrm{Kg} \mathrm{N} / \mathrm{ha}$ & $8.07 \pm 0.30^{\mathrm{c}}$ & $0.83 \pm 0.10^{\mathrm{ab}}$ & $46.7 \pm 3.05^{\mathrm{a}}$ & 2.4 \\
\hline $\mathrm{ZA}-120 \mathrm{Kg} \mathrm{N} / \mathrm{ha}$ & $8.44 \pm 0.25^{\mathrm{c}}$ & $0.57 \pm 0.0^{\mathrm{d}}$ & $42.3 \pm 2.50^{\mathrm{e}}$ & 1.6 \\
\hline $\mathrm{ZA}-150 \mathrm{Kg}$ N/ha & $9.58 \pm 0.55^{\mathrm{ab}}$ & $0.59 \pm 0.01^{\mathrm{d}}$ & $44.9 \pm 2.88^{b c}$ & 2.5 \\
\hline ZA -180 Kg N/ha & $9.59 \pm 0.61^{\mathrm{ab}}$ & $0.70 \pm 0.08^{\mathrm{bc}}$ & $41.1 \pm 0.56^{\mathrm{ef}}$ & 1.9 \\
\hline ZA - $210 \mathrm{Kg} \mathrm{N} / \mathrm{ha}$ & $9.99 \pm 0.22^{\mathrm{ab}}$ & $0.77 \pm 0.0^{\mathrm{b}}$ & $43.1 \pm 1.74^{\mathrm{d}}$ & 1.8 \\
\hline Average & 9.37 & 0.76 & 44.0 & 2.0 \\
\hline DMRT 5\% & NS & NS & NS & $\mathbf{S}$ \\
\hline $\mathrm{CV}(\%)$ & 12.31 & 13.90 & 3.58 & 21.91 \\
\hline
\end{tabular}

Data are mean of five plants; \pm Standard Error of mean; Values without common letters differ significantly at LSD P $<0.05$; Non Significant (NS) value on Duncan Multiple Range Test (DMRT) was computed at the 5\% probability level

Table 4. Effect of $\mathrm{N}$ fertilizer on plant population, grain yield and yield components of soybean in rice-soybean-soybean cropping system.

\begin{tabular}{|c|c|c|c|c|}
\hline Nitrogen Source and its dose & Number of Plants/ ha & Grain yield (t/ha) & $\begin{array}{c}\text { Number of Filled pods } \\
\text { /plant }\end{array}$ & $\begin{array}{l}\text { weight of } 100 \\
\text { seed }(\mathrm{g})\end{array}$ \\
\hline Without NS (Control) & $415.10 \pm 10.50^{\mathrm{d}}$ & $2.78 \pm 0.95^{\mathrm{d}}$ & $44.4 \pm 2.98^{\mathrm{bc}}$ & $10.45 \pm 0.89$ \\
\hline Without N (Control) & $460.94 \pm 20.25^{\mathrm{a}}$ & $2.75 \pm 0.45^{\mathrm{d}}$ & $35.0 \pm 3.56^{\mathrm{d}}$ & $10.85 \pm 1.03$ \\
\hline Urea $-30 \mathrm{Kg} \mathrm{N} / \mathrm{ha}$ & $447.92 \pm 14.33^{\mathrm{ab}}$ & $2.85 \pm 0.87^{\mathrm{c}}$ & $37.7 \pm 1.20^{\mathrm{d}}$ & $10.32 \pm 1.75$ \\
\hline Urea $-60 \mathrm{Kg} \mathrm{N} / \mathrm{ha}$ & $447.40 \pm 17.63^{\mathrm{ab}}$ & $2.85 \pm 0.65^{\mathrm{c}}$ & $48.5 \pm 2.96^{\mathrm{ab}}$ & $10.45 \pm 0.85$ \\
\hline Urea $-90 \mathrm{Kg} \mathrm{N} / \mathrm{ha}$ & $451.56 \pm 21.22^{\mathrm{a}}$ & $3.03 \pm 0.99^{\mathrm{b}}$ & $41.9 \pm 3.51^{\mathrm{c}}$ & $10.76 \pm 0.25$ \\
\hline Urea $-120 \mathrm{Kg} \mathrm{N} / \mathrm{ha}$ & $442.19 \pm 5.98^{\mathrm{b}}$ & $3.21 \pm 0.85^{\mathrm{a}}$ & $43.5 \pm 2.22^{\mathrm{bc}}$ & $10.57 \pm 0.62$ \\
\hline Urea $-150 \mathrm{Kg} \mathrm{N} / \mathrm{ha}$ & $460.94 \pm 14.36^{\mathrm{a}}$ & $3.12 \pm 0.67^{\mathrm{ab}}$ & $44.1 \pm 3.69^{\mathrm{bc}}$ & $10.48 \pm 0.56$ \\
\hline Urea $-180 \mathrm{Kg} \mathrm{N} / \mathrm{ha}$ & $442.19 \pm 09.52^{b}$ & $3.10 \pm 0.24^{\mathrm{ab}}$ & $50.8 \pm 2.52^{\mathrm{a}}$ & $10.32 \pm 0.99$ \\
\hline Urea $-210 \mathrm{Kg} \mathrm{N} / \mathrm{ha}$ & $451.04 \pm 18.87^{\mathrm{a}}$ & $2.86 \pm 0.20^{\mathrm{c}}$ & $50.3 \pm 1.78^{\mathrm{a}}$ & $10.31 \pm 1.02$ \\
\hline ZA - $30 \mathrm{Kg} \mathrm{N} / \mathrm{ha}$ & $457.81 \pm 17.85^{\mathrm{a}}$ & $3.02 \pm 0.50^{\mathrm{b}}$ & $44.5 \pm 1.00^{\mathrm{bc}}$ & $10.24 \pm 0.55$ \\
\hline ZA -60 Kg N/ha & $425.00 \pm 15.54^{\mathrm{c}}$ & $3.10 \pm 0.87^{\mathrm{ab}}$ & $48.7 \pm 2.50^{\mathrm{ab}}$ & $10.80 \pm 0.85$ \\
\hline ZA $-90 \mathrm{Kg} \mathrm{N} / \mathrm{ha}$ & $445.31 \pm 12.63^{\mathrm{ab}}$ & $2.84 \pm 0.91^{\mathrm{c}}$ & $48.8 \pm 2.47^{\mathrm{ab}}$ & $10.34 \pm 0.61$ \\
\hline $\mathrm{ZA}-120 \mathrm{Kg} \mathrm{N} / \mathrm{ha}$ & $458.85 \pm 24.65^{\mathrm{a}}$ & $3.06 \pm 0.84^{\mathrm{a}}$ & $42.8 \pm 2.85^{\mathrm{c}}$ & $10.31 \pm 0.63$ \\
\hline $\mathrm{ZA}-150 \mathrm{Kg}$ N/ha & $445.83 \pm 16.66^{\mathrm{ab}}$ & $3.16 \pm 0.34^{\mathrm{a}}$ & $46.5 \pm 3.57^{b}$ & $10.65 \pm 0.00$ \\
\hline $\mathrm{ZA}-180 \mathrm{Kg} \mathrm{N} / \mathrm{ha}$ & $458.33 \pm 21.03^{\mathrm{a}}$ & $3.10 \pm 0.87^{\mathrm{ab}}$ & $42.7 \pm 2.22^{\mathrm{c}}$ & $10.78 \pm 1.00$ \\
\hline $\mathrm{ZA}-210 \mathrm{Kg} \mathrm{N} / \mathrm{ha}$ & $447.39 \pm 14.52^{\mathrm{ab}}$ & $3.07 \pm 0.56^{\mathrm{ab}}$ & $42.2 \pm 1.98^{\mathrm{c}}$ & $10.99 \pm 0.49$ \\
\hline Average & 447.363 & 2.99 & 44.5 & 10.54 \\
\hline DMRT 5\% & $\mathrm{S}$ & NS & $\mathrm{S}$ & NS \\
\hline $\mathrm{CV}(\%)$ & 3.27 & 6.51 & 9.15 & 3.31 \\
\hline
\end{tabular}

Data are mean of five plants; \pm Standard Error of mean; Values without common letters differ significantly at LSD P $<0.05$; Non Significant (NS) value on Duncan Multiple Range Test (DMRT) was computed at the 5\% probability level 
Table 5 Effect of $\mathrm{N}$ fertilizer on vegetative growth of soybean in rice-rice-soybean cropping system.

\begin{tabular}{|c|c|c|c|c|}
\hline \multirow[t]{2}{*}{ Nitrogen Source and its dose } & \multicolumn{2}{|c|}{ Dry weight (g/plant) } & \multirow[t]{2}{*}{ Plant height (cm) } & \multirow{2}{*}{$\begin{array}{l}\text { Branches } \\
\text { (no./plant) }\end{array}$} \\
\hline & Shoot & Root & & \\
\hline Without NS (Control) & $8.51 \pm 1.56^{\mathrm{c}}$ & $0.94 \pm 0.08^{\mathrm{ab}}$ & $64.7 \pm 2.33^{\mathrm{d}}$ & $3.5 \pm 0.98$ \\
\hline Without N (Control) & $8.40 \pm 1.89^{\mathrm{c}}$ & $0.98 \pm 0.10^{\mathrm{a}}$ & $67.7 \pm 4.56^{\mathrm{c}}$ & $3.8 \pm 0.85^{\mathrm{ab}}$ \\
\hline Urea $-30 \mathrm{Kg} \mathrm{N} / \mathrm{ha}$ & $8.65 \pm 1.88^{\mathrm{c}}$ & $0.85 \pm 0.20^{\mathrm{bc}}$ & $66.5 \pm 2.05^{\mathrm{cd}}$ & $4.2 \pm 0.45^{\mathrm{a}}$ \\
\hline Urea $-60 \mathrm{Kg} \mathrm{N} / \mathrm{ha}$ & $7.19 \pm 1.26^{\mathrm{d}}$ & $0.85 \pm 0.17^{\mathrm{bc}}$ & $67.2 \pm 3.26^{\mathrm{c}}$ & $4.1 \pm 0.91^{\mathrm{ab}}$ \\
\hline Urea $-90 \mathrm{Kg} \mathrm{N} / \mathrm{ha}$ & $9.63 \pm 1.32^{\mathrm{b}}$ & $1.01 \pm 0.09^{\mathrm{a}}$ & $68.1 \pm 1.55^{\mathrm{b}}$ & $3.7 \pm 1.00^{\mathrm{b}}$ \\
\hline Urea $-120 \mathrm{Kg} \mathrm{N} / \mathrm{ha}$ & $9.88 \pm 0.99^{b}$ & $0.93 \pm 0.25^{\mathrm{ab}}$ & $67.9 \pm 2.10^{\mathrm{bc}}$ & $3.8 \pm 1.03^{\mathrm{ab}}$ \\
\hline Urea $-150 \mathrm{Kg} \mathrm{N} / \mathrm{ha}$ & $9.07 \pm 0.85^{\mathrm{bc}}$ & $0.95 \pm 0.15^{\mathrm{ab}}$ & $68.9 \pm 1.56^{\mathrm{ab}}$ & $4.1 \pm 0.99^{\mathrm{ab}}$ \\
\hline Urea $-180 \mathrm{Kg} \mathrm{N} / \mathrm{ha}$ & $8.90 \pm 1.45^{\mathrm{bc}}$ & $0.94 \pm 0.19^{\mathrm{ab}}$ & $62.1 \pm 3.05^{\mathrm{e}}$ & $3.3 \pm 0.74^{\mathrm{c}}$ \\
\hline Urea $-210 \mathrm{Kg}$ N/ha & $9.42 \pm 1.69^{\mathrm{b}}$ & $0.90 \pm 0.05^{\mathrm{b}}$ & $68.1 \pm 2.89^{b}$ & $3.3 \pm 0.52^{\mathrm{c}}$ \\
\hline ZA - $30 \mathrm{Kg} \mathrm{N} / \mathrm{ha}$ & $8.67 \pm 1.87^{\mathrm{c}}$ & $0.87 \pm 0.10^{\mathrm{b}}$ & $65.5 \pm 1.45^{\mathrm{cd}}$ & $3.9 \pm 0.98^{\mathrm{ab}}$ \\
\hline ZA -60 Kg N/ha & $9.73 \pm 2.06^{\mathrm{b}}$ & $1.01 \pm 0.02^{\mathrm{a}}$ & $69.2 \pm 2.22^{\mathrm{a}}$ & $3.6 \pm 1.00^{\mathrm{b}}$ \\
\hline ZA - $90 \mathrm{Kg} \mathrm{N} / \mathrm{ha}$ & $8.98 \pm 1.42^{\mathrm{bc}}$ & $0.89 \pm 0.16^{\mathrm{b}}$ & $69.9 \pm 1.74^{\mathrm{a}}$ & $3.4 \pm 0.75^{\mathrm{bc}}$ \\
\hline $\mathrm{ZA}-120 \mathrm{Kg} \mathrm{N} / \mathrm{ha}$ & $7.89 \pm 0.81^{\mathrm{dc}}$ & $0.80 \pm 0.23^{\mathrm{c}}$ & $65.6 \pm 2.56^{\mathrm{cd}}$ & $4.0 \pm 1.08^{\mathrm{ab}}$ \\
\hline $\mathrm{ZA}-150 \mathrm{Kg} \mathrm{N} / \mathrm{ha}$ & $9.61 \pm 0.45^{\mathrm{b}}$ & $1.00 \pm 0.25^{\mathrm{a}}$ & $68.5 \pm 3.00^{\mathrm{b}}$ & $4.0 \pm 0.82^{\mathrm{ab}}$ \\
\hline ZA - $180 \mathrm{Kg} \mathrm{N} / \mathrm{ha}$ & $9.84 \pm 1.56^{\mathrm{b}}$ & $0.97 \pm 0.13^{\mathrm{a}}$ & $65.0 \pm 2.15^{\mathrm{cd}}$ & $4.0 \pm 0.75^{\mathrm{ab}}$ \\
\hline ZA $-210 \mathrm{Kg} \mathrm{N} / \mathrm{ha}$ & $10.28 \pm 1.56^{\mathrm{a}}$ & $1.00 \pm 0.09^{\mathrm{a}}$ & $69.4 \pm 2.77^{\mathrm{a}}$ & $4.6 \pm 1.10^{\mathrm{a}}$ \\
\hline Average & 9.04 & 0.93 & 67.1 & 3.8 \\
\hline DMRT 5\% & $\mathrm{S}$ & $\mathrm{S}$ & $\mathrm{S}$ & $\mathrm{S}$ \\
\hline CV (\%) & 13.85 & 14.85 & 6.35 & 12.97 \\
\hline
\end{tabular}

Data are mean of five plants; \pm Standard Error of mean; Values without common letters differ significantly at LSD P $<0.05$; Non Significant (NS) value on Duncan Multiple Range Test (DMRT) was computed at the 5\% probability level

Table 6 Effect of $\mathrm{N}$ fertilizer on plant population, grain yield and yield components of soybean in rice-rice-soybean cropping system.

\begin{tabular}{|c|c|c|c|c|}
\hline $\begin{array}{l}\text { Nitrogen Source and its } \\
\text { dose }\end{array}$ & Number of Plants/ha & Grain yield (t/ha) & $\begin{array}{c}\text { Number of Filled } \\
\text { pods/plant }\end{array}$ & $\begin{array}{l}\text { weight of } 100 \text { seed } \\
\text { (g) }\end{array}$ \\
\hline Without NS (Control) & $480.90 \pm 10.25^{\mathrm{b}}$ & $2.84 \pm 0.85^{\mathrm{c}}$ & $40.2 \pm 2.00^{\mathrm{c}}$ & $10.16 \pm 1.02$ \\
\hline Without N (Control) & $492.36 \pm 12.02^{\mathrm{a}}$ & $2.84 \pm 0.26^{\mathrm{c}}$ & $42.8 \pm 1.85^{\mathrm{b}}$ & $10.40 \pm 1.40$ \\
\hline Urea $-30 \mathrm{Kg} \mathrm{N} / \mathrm{ha}$ & $492.36 \pm 15.62^{\mathrm{a}}$ & $3.42 \pm 0.74^{\mathrm{a}}$ & $41.6 \pm 3.05^{\mathrm{bc}}$ & $10.23 \pm 1.52$ \\
\hline Urea $-60 \mathrm{Kg} N / \mathrm{ha}$ & $493.06 \pm 20.23^{\mathrm{a}}$ & $3.11 \pm 0.62^{\mathrm{ab}}$ & $36.3 \pm 1.80^{\mathrm{d}}$ & $10.33 \pm 0.90$ \\
\hline Urea $-90 \mathrm{Kg} \mathrm{N} / \mathrm{ha}$ & $496.18 \pm 10.28^{\mathrm{a}}$ & $3.34 \pm 1.0^{\mathrm{a}}$ & $43.1 \pm 2.20^{\mathrm{b}}$ & $10.44 \pm 0.80$ \\
\hline Urea $-120 \mathrm{Kg} \mathrm{N} / \mathrm{ha}$ & $494.10 \pm 8.00^{\mathrm{a}}$ & $3.26 \pm 1.04^{\mathrm{ab}}$ & $41.0 \pm 2.45^{\mathrm{bc}}$ & $10.56 \pm 1.40$ \\
\hline Urea $-150 \mathrm{Kg} \mathrm{N} / \mathrm{ha}$ & $495.49 \pm 13.65^{\mathrm{a}}$ & $3.16 \pm 0.82^{\mathrm{ab}}$ & $42.8 \pm 2.40^{\mathrm{b}}$ & $10.38 \pm 1.84$ \\
\hline Urea $-180 \mathrm{Kg} \mathrm{N} / \mathrm{ha}$ & $495.49 \pm 19.40^{\mathrm{a}}$ & $3.20 \pm 0.60^{\mathrm{ab}}$ & $38.9 \pm 1.80^{\mathrm{cd}}$ & $10.35 \pm 0.90$ \\
\hline Urea $-210 \mathrm{Kg} \mathrm{N} / \mathrm{ha}$ & $495.14 \pm 25.30^{\mathrm{a}}$ & $2.93 \pm 0.75^{\mathrm{b}}$ & $39.0 \pm 2.00^{\text {cd }}$ & $10.27 \pm 1.42$ \\
\hline ZA -30 Kg N/ha & $495.49 \pm 6.00^{\mathrm{a}}$ & $3.09 \pm 1.00^{\mathrm{ab}}$ & $41.9 \pm 2.05^{\mathrm{bc}}$ & $10.47 \pm 0.90$ \\
\hline ZA -60 Kg N/ha & $490.63 \pm 7.50^{\mathrm{ab}}$ & $3.28 \pm 0.38^{\mathrm{ab}}$ & $40.2 \pm 1.80^{\mathrm{c}}$ & $10.38 \pm 1.20$ \\
\hline ZA $-90 \mathrm{Kg} \mathrm{N} / \mathrm{ha}$ & $490.28 \pm 9.20^{\mathrm{ab}}$ & $3.38 \pm 1.00^{\mathrm{a}}$ & $42.6 \pm 1.45^{\mathrm{b}}$ & $10.45 \pm 1.35$ \\
\hline $\mathrm{ZA}-120 \mathrm{Kg} \mathrm{N} / \mathrm{ha}$ & $495.49 \pm 14.20^{\mathrm{a}}$ & $2.91 \pm 0.74^{\mathrm{b}}$ & $40.9 \pm 2.70^{\mathrm{c}}$ & $10.28 \pm 1.80$ \\
\hline $\mathrm{ZA}-150 \mathrm{Kg} \mathrm{N} / \mathrm{ha}$ & $491.32 \pm 17.20^{\mathrm{a}}$ & $2.84 \pm 0.90^{\mathrm{c}}$ & $40.6 \pm 2.20^{\mathrm{c}}$ & $10.38 \pm 0.50$ \\
\hline $\mathrm{ZA}-180 \mathrm{Kg} \mathrm{N} / \mathrm{ha}$ & $491.32 \pm 14.20^{\mathrm{a}}$ & $3.22 \pm 0.42^{\mathrm{ab}}$ & $43.8 \pm 2.84^{\mathrm{b}}$ & $10.43 \pm 1.00$ \\
\hline $\mathrm{ZA}-210 \mathrm{Kg} \mathrm{N} / \mathrm{ha}$ & $494.79 \pm 15.10^{\mathrm{a}}$ & $3.32 \pm 0.98^{\mathrm{a}}$ & $49.7 \pm 3.00^{\mathrm{a}}$ & $10.36 \pm 2.00$ \\
\hline Average & 492.773 & 3.13 & 41.6 & 10.36 \\
\hline DMRT 5\% & NS & $\mathrm{S}$ & $\mathrm{S}$ & NS \\
\hline CV (\%) & 1.19 & 7.03 & 12.76 & 0.85 \\
\hline
\end{tabular}

Data are mean of five plants; \pm Standard Error of mean; Values without common letters differ significantly at LSD P $<0.05$; Non Significant (NS) value on Duncan Multiple Range Test (DMRT) was computed at the 5\% probability level 
Table 7 Effect of $\mathrm{N}$ fertilizer on macro nutrients uptake at $60 \mathrm{DAP}$ on soybean in rice-soybean-soybean cropping system.

\begin{tabular}{|lccccccc|}
\hline Nitrogen Source and its dose & $\mathbf{N}$ (g/plant) & $\mathbf{P}$ (g/plant) & $\mathbf{K}$ (g/plant) & $\mathbf{C a}(\mathrm{g} / \mathrm{plant})$ & $\mathbf{M g}(\mathrm{g} / \mathrm{plant})$ & $\mathbf{S O}_{4}(\mathrm{~g} / \mathrm{plant})$ \\
\hline Without NS (Control) & 0.2123 & 0.0291 & 0.1609 & 0.1489 & 0.0317 & 0.1002 \\
\hline Without N (Control) & 0.1814 & 0.0296 & 0.1446 & 0.1212 & 0.0386 & 0.1024 \\
\hline Urea $-30 \mathrm{Kg} \mathrm{N} / \mathrm{ha}$ & 0.2637 & 0.0291 & 0.1464 & 0.1367 & 0.0335 & 0.0926 \\
\hline Urea $-60 \mathrm{Kg} \mathrm{N} / \mathrm{ha}$ & 0.2342 & 0.0306 & 0.1635 & 0.1262 & 0.0373 & 0.1147 \\
\hline Urea $-90 \mathrm{Kg} \mathrm{N} / \mathrm{ha}$ & 0.2117 & 0.0301 & 0.1651 & 0.1272 & 0.0534 & 0.1049 \\
\hline Urea $-120 \mathrm{Kg} \mathrm{N} / \mathrm{ha}$ & 0.1995 & 0.0292 & 0.1615 & 0.0808 & 0.0379 & 0.0963 \\
\hline Urea $-150 \mathrm{Kg} \mathrm{N} / \mathrm{ha}$ & 0.1745 & 0.0328 & 0.1610 & 0.1379 & 0.0415 & 0.1070 \\
\hline Urea $-180 \mathrm{Kg} \mathrm{N} / \mathrm{ha}$ & 0.2345 & 0.0299 & 0.1657 & 0.1387 & 0.0459 & 0.0699 \\
\hline Urea $-210 \mathrm{Kg} \mathrm{N} / \mathrm{ha}$ & 0.2025 & 0.0274 & 0.1541 & 0.1259 & 0.0365 & 0.0912 \\
\hline ZA -30 Kg N/ha & 0.2563 & 0.0336 & 0.1780 & 0.1048 & 0.0488 & 0.1048 \\
\hline ZA -60 Kg N/ha & 0.2275 & 0.0329 & 0.1694 & 0.1578 & 0.0445 & 0.0958 \\
\hline ZA -90 Kg N/ha & 0.1816 & 0.0202 & 0.1380 & 0.1122 & 0.0395 & 0.0678 \\
\hline ZA -120 Kg N/ha & 0.2009 & 0.0287 & 0.1401 & 0.0996 & 0.0405 & 0.0751 \\
\hline ZA -150 Kg N/ha & 0.2347 & 0.0307 & 0.1801 & 0.1284 & 0.0441 & 0.1092 \\
\hline ZA -180 Kg N/ha & 0.2282 & 0.0307 & 0.1774 & 0.1247 & 0.0432 & 0.1026 \\
\hline ZA -210 Kg N/ha & 0.2547 & 0.0330 & 0.1848 & 0.1229 & 0.0490 & 0.1139 \\
\hline Average & 0.2186 & 0.0298 & 0.1619 & 0.1246 & 0.0416 & 0.0968 \\
\hline
\end{tabular}

Table 8 Effect of $\mathrm{N}$ fertilizer on macro nutrients uptake at 60 DAP by soybean grown in rice-rice-soybean cropping system.

\begin{tabular}{|c|c|c|c|c|c|c|}
\hline Nitrogen Source and its dose & $\mathbf{N}$ (g/plant) & P (g/plant) & $\mathrm{K}$ (g/plant) & Ca (g/plant) & Mg (g/plant) & $\begin{array}{c}\mathrm{SO}_{4} \\
\text { (g/plant) }\end{array}$ \\
\hline Without NS (Control) & 0.1889 & 0.0204 & 0.1396 & 0.0962 & 0.0340 & 0.0647 \\
\hline Without N (Control) & 0.2260 & 0.0244 & 0.1579 & 0.1058 & 0.0370 & 0.0983 \\
\hline Urea $-30 \mathrm{Kg}$ N/ha & 0.1981 & 0.0225 & 0.1600 & 0.0848 & 0.0320 & 0.0822 \\
\hline Urea $-60 \mathrm{Kg} \mathrm{N} / \mathrm{ha}$ & 0.1740 & 0.0209 & 0.1345 & 0.1057 & 0.0324 & 0.0690 \\
\hline Urea $-90 \mathrm{Kg} \mathrm{N} / \mathrm{ha}$ & 0.2302 & 0.0270 & 0.1820 & 0.1425 & 0.0482 & 0.1059 \\
\hline Urea $-120 \mathrm{Kg} \mathrm{N} / \mathrm{ha}$ & 0.2154 & 0.0277 & 0.1808 & 0.0968 & 0.0405 & 0.0860 \\
\hline Urea $-150 \mathrm{Kg} \mathrm{N} / \mathrm{ha}$ & 0.2231 & 0.0281 & 0.1723 & 0.0898 & 0.0435 & 0.0970 \\
\hline Urea $-180 \mathrm{Kg} \mathrm{N} / \mathrm{ha}$ & 0.1949 & 0.0240 & 0.1673 & 0.1157 & 0.0329 & 0.0961 \\
\hline Urea $-210 \mathrm{Kg} \mathrm{N} / \mathrm{ha}$ & 0.2251 & 0.0245 & 0.1752 & 0.0980 & 0.0424 & 0.1017 \\
\hline ZA -30 Kg N/ha & 0.2332 & 0.0234 & 0.1465 & 0.1049 & 0.0347 & 0.0858 \\
\hline ZA - $60 \mathrm{Kg} \mathrm{N} / \mathrm{ha}$ & 0.2452 & 0.0292 & 0.1761 & 0.1275 & 0.0409 & 0.1080 \\
\hline ZA -90 Kg N/ha & 0.2263 & 0.0233 & 0.1572 & 0.1060 & 0.0350 & 0.0790 \\
\hline $\mathrm{ZA}-120 \mathrm{Kg} \mathrm{N} / \mathrm{ha}$ & 0.2091 & 0.0213 & 0.1452 & 0.0931 & 0.0323 & 0.0813 \\
\hline $\mathrm{ZA}-150 \mathrm{Kg} \mathrm{N} / \mathrm{ha}$ & 0.2508 & 0.0288 & 0.1682 & 0.1086 & 0.0394 & 0.1105 \\
\hline ZA $-180 \mathrm{Kg} \mathrm{N} / \mathrm{ha}$ & 0.2637 & 0.0276 & 0.1732 & 0.1260 & 0.0374 & 0.0964 \\
\hline $\mathrm{ZA}-210 \mathrm{Kg} \mathrm{N} / \mathrm{ha}$ & 0.3290 & 0.0370 & 0.1789 & 0.1470 & 0.0473 & 0.0894 \\
\hline Average & 0.2271 & 0.0256 & 0.1634 & 0.1093 & 0.0381 & 0.0907 \\
\hline
\end{tabular}

Application of $\mathrm{N}$ fertilizers caused increases in the soybean grain yield and weight of 100 seeds and it is significantly differ than the control. Soybean grain yield and weight of 100 seed were higher and reached an average of $2.99 \mathrm{t} /$ ha and $10.54 \mathrm{~g}$ respectively (Table 4). Similarly number of pod was also reported at par or higher than the control and it reach up to $50.8 \mathrm{pod} / \mathrm{plant}$ on the application of urea at $180 \mathrm{Kg} \mathrm{N} / \mathrm{ha}$. Result of the study revealed that Application of urea shows superiority over the application of ZA (Table 4). While the other doses of $\mathrm{N}$ fertilizers did not show significant increases the number of pods as compared to the dose of $180 \mathrm{~kg} / \mathrm{ha}$. Lack of crop response to fertilizer $\mathrm{N}$ presumably related with $\mathrm{N}$ fixation of indigenous Rhizobium. Generally the soil frequently used for soybeans plantation contain enough Rhizobium population. According to Salvagiotti et al. (2008) a negative exponential relationship was observed between $\mathrm{N}$ fertilizer rate and $\mathrm{N} 2$ fixation when $\mathrm{N}$ was applied in the top $0-20 \mathrm{~cm}$ of soil or on the soil surface. They reported highest $\mathrm{N}$ fixation (337 $\mathrm{kg} / \mathrm{ha}$ ) in the treatment without chemical $\mathrm{N}$ fertilization application and a consequence exponential decline 129 and 17 $\mathrm{kg} / \mathrm{ha}$ was reported in maximum $\mathrm{N} 2$ fixation at the application of 100 and $300 \mathrm{~kg} / \mathrm{ha}$ respectively. 
Result of the study clearly suggested that cropping pattern having soybean after rice shows better soybean growth than the soybean after soybean. The average plant height reached 67.1 $\mathrm{cm}$, while the average shoot weight was reported $9.04 \mathrm{~g} / \mathrm{plant}$ and it was higher than the control (Table 5). However shoot dry weight, plant height and number of branches was not affected by $\mathrm{N}$ fertilizer. Among the two nitrogen sources ZA shows superiority over the urea but it is not significantly differ. With respect to the response of $\mathrm{N}$ fertilizer, addition of sulfur powder@282 kg / ha (to equalize the content of $\mathrm{S}$ in the treatment of $210 \mathrm{~kg} \mathrm{~N} / \mathrm{ha}$ of ZA) in the treatment without $\mathrm{N}$ increased the weight of 100 -seeds from 10.16 to $10.40 \mathrm{~g}$. Combination of $\mathrm{N}$ and $\mathrm{S}$ fertilizers did not have any significant effect on the weight of 100 seeds. Similarly, $\mathrm{N}$ fertilizer did not increase number of pods and weight of seeds per plant (Table 6). The positive influence of sulfur on the weight of 100 seeds might be related to its role in seed production and quality especially in the formation of seed proteins (Jamal et al., 2010; Devi et al., 2012; Choudhary et al., 2014).

Nitrogen fertilization had no effect on grain yield in case of grown soybean after soybean while a positive effect on grain yield was obtained in the condition of grown soybean after rice. Grain yield of soybean grown after rice without $\mathrm{N}$ fertilizer was $2.84 \mathrm{t} / \mathrm{ha}$. The application of urea at $30 \mathrm{~kg} / \mathrm{ha}$ increased grain yield by $0.58 \mathrm{t} / \mathrm{ha}(20 \%)$ to $3.42 \mathrm{t} / \mathrm{ha}$, and it was the highest grain yield level while the using of ZA @ 90 $\mathrm{kg} / \mathrm{ha}$ increased the grain yield by $0.54 \mathrm{t} / \mathrm{ha}(19 \%)$ to $3.38 \mathrm{t} /$ ha (Table 6). These findings were different than the findings of Kuntyastuti et al. (2007) those have reported that $\mathrm{N}$ fertilization@30-210 kg / ha in clayey loam textured soils poor of $\mathrm{N}$ did not increase grain yield of soybean grown after rice (rice-rice-soybean) as well as after soybean (rice-soybeansoybean).

The results of this study add to the fact that response of soybean to fertilizer $\mathrm{N}$ has been inconsistent (Gan et al., 2003; Barker \& Sawyer, 2005) which may be related to large variation in indigenous soil $\mathrm{N}$ supply such as net soil $\mathrm{N}$ mineralization, irrigation, atmospheric deposition, or other factors affecting yield responses to $\mathrm{N}$ (Salvagiotti et al., 2008). Although growth and grain yield of soybean grown after rice was better than soybean grown after soybean but the $\mathrm{N}$ uptake in both fields were relatively similar, as well as other nutrients (Table 7 and 8). This indicates that cropping pattern had no effect on nutrient uptake by soybean plants (Table 1).

\section{Conclusion}

Soybean planted after rice in rice-rice-soybean cropping pattern required $30 \mathrm{~kg} \mathrm{~N} /$ ha of urea or $90 \mathrm{~kg} \mathrm{~N} /$ ha of ZA to increase grain yield by $0.5 \mathrm{t} /$ ha while soybean planted after soybean in rice-soybean-soybean cropping pattern did not require $\mathrm{N}$ fertilizer.
The authors thank Prof. Sudaryono in the Indonesian Legume and Tuber Crops Institute (ILETRI), for his valuable comments on the manuscript

\section{Conflict of interest}

Authors would hereby like to declare that there is no conflict of interests that could possibly arise.

\section{References}

Adisarwanto T, Kuntyastuti H, Suhartina (1998) The efficiency of fertilization using soil testing and soybeans plant on several types of wetland soil. The Increase in Input Use Efficiency, Resources and Productivity of Soybean. Balitkabi Technical Report 1997/1998 Pp 1-19.

Adisarwanto T, Riwanodja, Suhartina (1999) Study of soil and plant nutrient status and soybean yield in Entisol and Vertisol. Increasing the Efficiency of Fertilizer Use, Land Management and Soybeans Productivity. Balitkabi Technical Report 1998/1999 Pp 1-17

Adisarwanto T, Suhartina (2000) Determination of P fertilizer recommendation based on soil tests in Entisol. Increasing Efficiency of Nutrient, Water and Land Management Improvement Efficiency of Nutrient, Water and Land Management for Soybean in Entisol and Oxisol Paddy Fields. Balitkabi Final Report 1999/2000 Pp 1-15.

Adisarwanto T, Kuntyastuti H, Rahmianna AA, Riwanodja (2001) Optimization and efficiency of nutrient and organic matter management for soybean in paddy fields. In: Adisarwanto, Kuntyastuti H, Radjit BS, Manshuri AG, and Suryantini (Eds). The Optimization of Soil Physic and Crop Management and The Improvement of Soybean Seed yield Quality in Wetland and Dryland. Book II No. 1, Pp 6-56.

Al-Kaisi MM, Yin X, Licht MA (2005) Soil carbon and nitrogen changes as influenced by tillage and cropping systems in some Iowa soils. Agriculture. Ecosystems and Environment. 105:635-647. doi:10.1016/j.agee.2004.08.002.

Barker DW, Sawyer JE (2005) Nitrogen application to soybean at early reproductive development. Agronomy Journal 97: $615-619$.

Choudhary P, Jhajharia A, Kumar R (2014) Influence of sulphur and zinc fertilization on yield, yield components and quality traits of soybean (Glycine Max L.). The Bioscan 9:137142

Devi KN, Singh LNK, Singh MS, Singh SB, Singh KK (2012) Influence of Sulphur and Boron fertilization on yield, quality, nutrient uptake and economics of Soybean (Glycine max) 
under upland conditions. Journal of Agricultural Science 4: 110. DOI: $10.5539 /$ jas.v4n4p1.

Dou F, Wright AL, Hons FM (2007) Depth distribution of soil organic $\mathrm{C}$ and $\mathrm{N}$ after long-term soybean cropping in Texas. Soil \& Tillage Research 94:530-536. doi:10.1016/j.still.2006.10.001

Gan, Y, Stulen I, van Keulen H, Kuiper PJC (2003) Effect of N fertilizer topdressing at various reproductive stages on growth, N2 fixation and yield of three soybean (Glycine max (L.) Merr.) genotypes. Field Crops Research 80: 147-155. doi:10.1016/S0378-4290(02)00171-5.

Gentile R, Vanlauwe B, Chivenge P, Six J (2008) Interactive effects from combining fertilizer and organic residue inputs on nitrogen transformations. Soil Biology and Biochemistry 40:2375-2384. doi:10.1016/j.soilbio.2008.05.018.

Gomez KA, Gomez AA (1984) Statistical Procedures for Agricultural Research $2^{\text {nd }}$ ed. New York. John Wiley \& Sons

Jamal A, Moon YS, Abdin MZ (2010) Sulphur -a general overview and interaction with nitrogen. Australian Journal of Crop Science 4:523-529

Kuntyastuti H, Wijanarko A, Soedarjo M, Manshuri AG (2007) Nitrogen fertilizer on soybean in rice-soybean and soybean-soybean cropping pattern. In: Harnowo, Rahmiana AA, Suharsono, Muchlish MA, Rozi F, Subandi, and Makraim AK (Eds) Increasing Production of Legumes and Tuber Crops Supporting Food independency. Proceedings of National Seminar. Pp: 243-254

Riwanodja, Purwaningrahayu RD, Adisarwanto T (2001) The efficiency of fertilizer $\mathrm{N}$ and $\mathrm{S}$ on soybean in paddy fields. Nutrient and Water Management to Improve Efficiency and Productivity of Soybean. Balitkabi Technical Report 2000.
Salvagiotti F, Cassman KG, Specht JE, Walters DT, Weiss A, Dobermann A (2008) Nitrogen uptake, fixation and response to fertilizer $\mathrm{N}$ in soybeans: A review. Field Crops Research108:1-13. doi: 10.1016/j.fcr.2008.03.001.

Santoso D, Suthipradit S, Sajjapongse A (2001) Soil nutrient status as an indicator of sustainable farming systems. In: Subagyo H, Sabiham S, Shofiyati R, Siswanto AB, Irawan, Rahman A, Ropiq S (Eds) Structuring the Use of Land As Devices of Spatial Planning In Order To Increase Welfare. Proceedings of the National Congress VI HITI, held on 12-15 December 1995 Jakarta, pp 225-231.

Spiertz JHJ (2010) Nitrogen, sustainable agriculture and food security. A review. Agronomy for Sustainable Development 30:43-55. doi: 10.1051/agro:2008064

Suryantini, Kuntyastuti H (1998) The use of Rhizoplus and Urea on soybean in rice-rice-soybean and rice-soybeansoybean cropping pattern. In: Sudaryono, Soedarjo M, Widodo Y, Suyamto H (Eds.). Proceedings of the National Seminar and Annual Meeting of the Regional Commissioner HITI 1998. Book II, Pp 80-86

Suryantini, Rahmiana AA (2001) Increasing the effectiveness of rhizoplus and inorganic fertilizer for soybean productivity in Regosol and Vertisol soil through the use of organic fertilizers. In: Arsyad DM, Soejitno J, Kasno A, Sudaryono (Eds.). Performance of Technology to Increase Productivity for Legume and Tuber Crops. Agricultural Research and Development Center Pp 269-277.

Suryantini (2008) Improvement of soybean yield in paddy fields planted after rice and after soybeans through the use of organic, inorganic and biological fertilizer. Agritek 17: 422428 